\title{
Depressão em idosos residentes em uma instituịạão asilar na cidade de Juazeiro do Norte, Ceará, Brasil
}

\section{|Depression in elderly residents in a nursing home North Juazeiro City, Ceara, Brazil}

\author{
Diego Coelho do Nascimento ${ }^{1}$ \\ Maria Adriana Calixto de Brito ${ }^{2}$ \\ Aurélio Dias Santos ${ }^{3}$
}

\section{Resumo}

O envelhecimento da população é um fenômeno crescente e pode ser verificado nos mais diversos países do globo. O Brasil acompanha essa tendência mundial, onde é perceptível a ampliação da população idosa brasileira e relacionado a esse fato, aumenta-se, proporcionalmente, o acometimento de doenças que atingem esse grupo específico. Entre elas, destaca-se a depressão, enfermidade mental mais frequente em pessoas da terceira idade. O presente estudo teve como objetivo principal verificar a prevalência de idosos com quadro depressivo residentes na casa do Idoso Instituição Asilar da cidade de Juazeiro do Norte/CE. A população do estudo constou de 40 idosos residentes na supracitada instituição asilar. O instrumento para a coleta de dados foi o questionário da escala de depressão geriátrica, adaptada de Yesavage et al. (1983), versão curta constando de 15 itens. Os dados foram analisados a partir da estatística descritiva através do EXCEL, 2010. Após a tabulação dos dados, os mesmos foram apresentados por meio de gráficos.

\section{Abstract}

The aging population is a growing phenomenon and can be found in various countries around the globe. Brazil is following this global trend which is noticeable expansion of the elderly population and related to this fact, it increases proportionally to involvement of the diseases that affect that particular group. Among them, there is depression, mental illness more common in the elderly. The present study aimed to determine the prevalence of depression in elderly home residents in the elderly - nursing home in the city of Juazeiro do Norte / CE. The study population consisted of 40 elderly nursing home residents in the above. The instrument for data collection was the questionnaire of the Geriatric Depression Scale, adapted from Yesavage et al. (1983), short version consisting of 15 items. Data were analyzed using descriptive statistics by EXCEL, 2007. After tabulating the data, they were presented through graphs.
Deseritores: Depressão, Prevalêneia, Idosos; Asilos

Keywords: Depression Prevalenee, Seniors, Nursing Homes

1 Baeharel em Fisioterapia, Lieeneiado em Geografia, Mestre em
Desenvolvimento Regional Sustentável e Professor da Universidade
Federal do Cariri - UFCA.

${ }^{2}$ Graduada em Fisioterapia e Letras, Pós-graduada em Fisiologia do Exercicio, Dermatofuncional, Educação de Jovens e Adultos e Gestão Eseolar.

${ }^{3}$ Bacharel em Fisioterapia, Pós-graduado em Doeêneia no Ensino Superior, Mestrando em Fisioterapia e Professor da Faculdade Leão Sampaio.

Para correspondência:

Diego Coelho do Nascimento

Email: diegocn_jua@yahoo.com.br 
Introdụão

O envelhecimento da população é um fenômeno verificado nos mais diversos países do globo. O Brasil acompanha essa tendência mundial, é perceptível o aumento desse grupo de indivíduos na pirâmide etária brasileira. A Organização Mundial de Saúde - OMS projeta que até 2020 o grupo de idosos corresponderá a $15 \%$ da população brasileira. Em 2025, estima-se que a população idosa deverá ter aumentado em até 15 vezes, enquanto a população total deverá ter crescido apenas 5 vezes$^{2}$.

Dessa forma, sendo a terceira idade a faixa etária que mais cresce no Brasil, proporcionalmente, as doenças decorrentes da velhice também terão acréscimos consideráveis e condizentes com o número total de idosos. Nesse contexto, destaca-se a prevalência de doenças neurológico-degenerativas e as tendências a depressão ${ }^{7}$.

Confirmando a gravidade desse agravo à saúde, é interessante destacar que "A depressão ocupa o quinto lugar como o problema de saúde mais prevalente no mundo, afetando 120 milhões de pessoas, segundo a OMS. De acordo com as projeções, estima-se que, em 20 anos a depressão ocupará o $2^{\circ}$ lugar, perdendo apenas para as doenças cardíacas ${ }^{6 "}$.

A depressão devido a sua multiplicidade de manifestações é de difícil conceituação e diagnóstico. Contudo, devido a ampliação do número de pesquisas e a inserção de novas formas de diagnóstico esse quadro vem mudando gradativamente, porém, ainda há muito a ser elucidado.

"A depressão pode ser definida como um transtorno de humor, que é um problema psicológico que se expressa através de uma ampla variedade de transtornos físicos e funcionais. $\mathrm{O}$ declínio progressivo do humor pode ser visto também do ponto de vista biológico ou orgânico, com as mudanças no sistema endócrino, neurológico e fisiológico ${ }^{1 "}$.

Complementando o conceito de depressão exposto acima, é importante destacar que "a depressão configura-se como um estado de alterações do humor envolvendo irritabilidade, tristeza profunda, apatia, disforia, perda da capacidade de sentir prazer e ainda, alterações cognitivas, motoras e somáticas. Ela difere de uma tristeza normal pela intensidade e duração prolongada dos sintomas, e tais sintomas interferem no funcionamento social do indivíduo, bem como em outras áreas significativas de sua vida, como: trabalho, relacionamentos amorosos ou amizades4."
O quadro depressivo em idosos se apresenta de maneira atípica ou indireta no que se refere à queixas múltiplas, geralmente, associadas a ansiedade. Nesse sentido, podem surgir alterações do sono e de apetite, sintomas psiquiátricos, perda da energia, sensação de culpa, tristeza subjetiva, diminuição da concentração e pensamentos sobre a morte ${ }^{7}$.

Quando acometido por depressão, o idoso sente-se angustiado, devido tanto aos sentimentos como as limitações físicas. Desencadeando, uma grande variedade de transtornos físicos e funcionais para esses indivíduos. Outros sintomas também podem ajudar no diagnostico de depressão em idosos, tais como: falta de apetite, baixa auto-estima, falta de iniciativa, déficit de concentração ${ }^{7}$.

O quadro depressivo em indivíduos da terceira idade é ainda mais grave quando a realidade é referente a instituições asilares ou instituições de longa permanência. Esta situação pode ser explicada através do seguinte ponto de vista: "O idoso que se encontra em Instituição de Longa Permanência, está separado do ambiente familiar e é levado a conviver com estranhos, muitas vezes isolado da atualidade cultural, além de estar experimentando a incômoda situação de abandono, dependência e inutilidade ${ }^{1} . "$

Complementando esses aspectos agravantes para o acometimento por depressão de idosos em instituições asilares, pode-se destacar também: o declínio físico continuado, a perda do status ocupacional e econômico e a maior frequência de doenças físicas ${ }^{7}$.

Diante do exposto, o presente trabalho se fez necessário para a ampliação dos estudos relacionados a depressão em indivíduos da terceira idade, partindo do aspectos supracitados. Sendo assim, o presente estudo visou identificar a prevalência de depressão em indivíduos idosos residentes em uma instituição asilar da cidade de Juazeiro do Norte - CE.

\section{Materiais e Método}

A presente pesquisa é do tipo descritiva, quantitativa, realizada na Casa do Idoso, entidade sem fins lucrativos localizada no Município de Juazeiro do Norte - Ceará que atende idosos desamparados do município e das cidades vizinhas. A instituição asilar recebeu uma carta 


\begin{tabular}{|c|c|c|}
\hline 1. Encontra-se, de uma forma geral, satisfeito com a vida? & SIM & $\mathrm{NA \tilde {O }}$ \\
\hline 2. Tem abandonado muitas de suas tarefas habituais e hobbies? & SIM & NÃO \\
\hline 3. Sente que a sua vida está vazia? & SIM & NÃO \\
\hline 4. Sente-se aborrecido frequentemente? & SIM & NÃO \\
\hline 5. Encontra-se bem disposto a maior parte do tempo? & SIM & NÃO \\
\hline 6. Teme que qualquer coisa de mal pode acontecer-lhe? & SIM & NÃO \\
\hline 7. Sente-se feliz a maior parte do tempo? & SIM & NÃO \\
\hline 8. Sente-se com frequência desemparado/a, desprotegido/a? & SIM & NÃO \\
\hline 9. Prefere ficar em casa, em vez de sair e fazer coisas novas? & SIM & NÃO \\
\hline 10. Acha que tem mais problemas de memória do que a maioria das pessoas? & SIM & NÃO \\
\hline 11. Neste momento, acha que é maravilhoso estar vivo? & SIM & $\mathrm{NÃO}$ \\
\hline 12. Sente-se útil atualmente? & SIM & NÃO \\
\hline 13. Sente-se cheio de energia? & SIM & $\mathrm{NÃO}$ \\
\hline 14. Sente-se sem esperança neste momento? & SIM & NÃO \\
\hline 15. Acha que a maioria das pessoas está em melhor situação do que a sua? & SIM & NÃO \\
\hline
\end{tabular}

Tabela 1. Questionário da Escola de Depressão Geriátrica, adaptado de Yesevage et al (1983), versão curta.

Fonte: Martinez et al (2002) 5

dos pesquisadores eméritos solicitando permissão para o desenvolvimento da pesquisa.

A população do estudo constou de 40 idosos residentes na supracitada instituição asilar com amostragem constituída de 24 mulheres $(60 \%)$ e 16 homens $(40 \%)$, com idades igual ou superior a 60 anos.

Essa amostra foi estabelecida a partir dos seguintes critérios de inclusão: idoso orientado quanto ao tempo e ao espaço; condição de interatividade com os pesquisadores e concordassem em responder o questionário da pesquisa. Os critérios de exclusão foram: idosos que não se encaixassem nos critérios de inclusão, ou seja, aqueles com diagnóstico prévio de déficit cognitivo com quadros que variam de demência a estado confusionais.

O instrumento para a coleta de dados foi o questionário da escala de depressão geriátrica, adaptada de Yesavage et al (1983), versão curta constando de 15 itens.

Vale ressaltar que, os entrevistadores foram treinados para realizarem a pesquisa e seguir os mesmos critérios. A aplicação do questionário ocorreu no período compreendido entre os meses de setembro e outubro de 2011.

Os dados foram analisados a partir da estatística descritiva através do EXCEL 2010. Após a tabulação dos dados, os mesmos foram apresentados por meio de gráficos.

\section{Resultadlos e Diseussão}

Na amostra total constituída por 40 idosos residentes na instituição asilar em questão, tivemos a proporção de 24 mulheres (60\%) e 16 mulheres (40\%), como demonstrado na figura 1.
Com relação ao acometimento ou não por depressão através da análise das respostas obtidas com o Questionário da Escala de Depressão Geriátrica, versão abreviada com 15 itens é importante destacar que a interpretação dos resultados se baseou nas recomendações dos autores do referido questionário. Cuja análise baseou-se nos escores obtidos: de 0 a 5 pontos exame normal; de 5 a 10 pontos - indícios de quadro depressivo leve e acima de 11 pontos provável depressão severa.

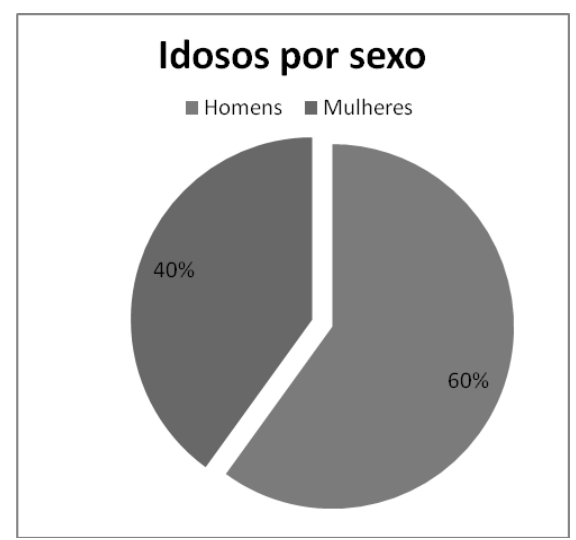

Gráfico 1. Distribuição da amostragem por sexo.

Fonte: Elaboração própria

Nesse sentido, constatou-se que $65 \%$ dos idosos da referente pesquisa possuem indícios de quadro depressivo, seja essa depressão leve ou severa. Referente a classificação, 14 indivíduos idosos (35\%) foram enquadrados como normais, 20 idosos (50\%) obtiveram escores que os encaixaram no quadro de depressão leve e 06 idosos $(15 \%)$ 
foram considerados como portadores de depressão leve.

\section{Prevalência do Quadro Depressivo}

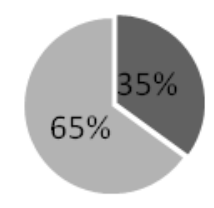

- Depressivos Não-Depressivos

Gráfico 2. Prevalência do quadro depressivo

Fonte: Elaboração própria

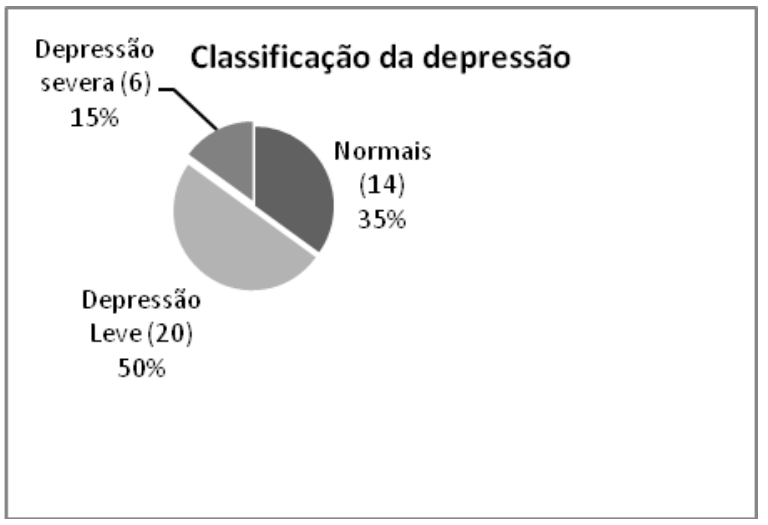

Gráfico 3. Classificação da depressão

Fonte: Elaboração própria

A prevalência obtida no estudo em questão vai de acordo com os resultados obtidos em pesquisas correlatas. A prevalência de depressão e transtornos depressivos nos idosos em geral oscila de 10 a 20$27 \% \%^{7}$. Quando se trata de idosos institucionalizados a taxa da prevalência de depressão se eleva numa variação de 25 a $85 \%$. Dessa forma, o presente estudo atingiu patamares similares a de pesquisas anteriores.

Essa diferença verificada entre os índices de depressão em idosos de maneira geral e idosos institucionalizados é verificada em função de diversos fatores. Entre eles temos os denominados estressores externos que irão influenciar no aparecimento de depressão em qualquer indivíduo idoso, tais como: saúde física alterada, morte de ente querido e isolamento social.

Com relação aos indivíduos da terceira idade institucionalizados, é necessário considerar que os mesmos são obrigados a se adaptar a uma rotina de horários, a dividir seu ambiente com desconhecidos, os mesmos têm que se acostumar com a ausência da família. Nesse contexto, os idosos passam a se ver como apenas mais um dentro da coletividade ${ }^{2}$.

\section{Conclusões}

Concluímos que a prevalência de depressão nos idosos estudados foi de $65 \%$ na Casa do Idoso. Onde do total geral obteve-se os seguintes números: 14 indivíduos idosos (35\%) foram enquadrados como normais 20 idosos (50\%) obtiveram escores que os encaixaram no quadro de depressão leve e 06 idosos (15\%) foram considerados como portadores de depressão leve.

Sendo assim, a maioria dos idosos da amostra que se encaixam no quadro depressivo, enquadram-se na classificação leve desta patologia. Portanto, há uma maior possibilidade de reversão desse quadro através de medidas estratégicas, como a identificação das causas da depressão e a identificação dos fatores de risco para a ocorrência desta, para a adoção de medidas específicas.

Nesse contexto, percebe-se que o idoso necessita de atividades multidisciplinares que possam preencher seu tempo de forma saudável, tanto física como mentalmente. Visando, dessa forma, evitar o aparecimento de doenças psicossomáticas, em especial, a depressão. Sendo que, esse quadro deve ser ainda mais analisado e aprofundado quando se refere a ocorrência de depressão em idosos institucionalizados. Já que, nesse grupo os índices de depressão podem chegar aos $80 \%$.

\section{Referências}

1. Andrade ACA, Lima FRA, Silva LFA, Santos SSC. Depressão em idosos de uma instituição de longa permanência (ILP): proposta de ação de enfermagem. Rev Gaúcha Enferm, Porto Alegre (RS) 2005 abr; 26(1):57-66.

2. Cheloni CFP, Pinheiro FLS, Filho MC, Medeiros AL. Prevalência de depressão em idosos institucionalizados no Município de Mossoró/RN 
segundo escala de depressão geriátrica (Yesavage). Universidade do Estado do Rio Grande do Norte UERN. Revista Expressão, Mossoró, 34 (1-2); p. 63-74; jandez. 2003.

3. Giavoni A, Melo GF, Parente I, Dantas G. Elaboração e validação da Escala de Depressão para Idosos. Cad. Saúde Pública, Rio de Janeiro, 24(5), mai, 2008.

4. Oliveira DAAP, Gomes L, Oliveira RF. Prevalência de depressão em idosos que frequentam centros de convivência. Rev. Saúde Pública, 2006: 49 (4).

5. Martinez J, Onis MC, Dueñas R, Albert C, Aguado C, Luque R. (2002). Versión española del cuestionario de Yesevage abreviado (GDS) para el despistaje de depresion em mayores de 65 años: adaptacion y validacion. Revista de Medicina Familiar y Comunitaria, 12, 620-630.

6. Pinho MX, Custódia O, Makdisse M. Incidência de depressão e fatores associados em idosos residentes na comunidade: revisão de literatura. Revista Brasileira de Geriatria e Gerontologia, 2009.

7. Silva GB, Silva VB, Lopes RC, Silva JWF. Caracterizando a depressão no idoso: Uma revisão bibliográfica. Enciclopédia biosfera, Centro Científico Conhecer - Goiânia, vol. 6. N. 9, 2010.

8. Sousa RL, Medeiros JG, Moura ACL, Souza CLM. Validade e fidedignidade da Escala de depressão geriátrica na identificação de idosos deprimidos em um hospital geral. Universidade Federal da Paraíba. J. Brasil Psiquiátrico, 56(2), 2007. 